Check for updates

Cite this: RSC Adv., 2019, 9, 30071

\title{
Formation of vesicles-in-a-vesicle with asymmetric lipid components using a pulsed-jet flow method
}

\author{
Koki Kamiya, ${ }^{\text {ac }}$ Toshihisa Osaki ${ }^{a}$ and Shoji Takeuchi (D) *ab
}

Lipid distribution in intracellular vesicles is different from that in the plasma membrane of eukaryotic cells. The lipid components in the intracellular vesicles are composed of phosphatidylserine and phosphatidylethanolamine in the outer leaflet and phosphatidylcholine and sphingomyelin in the inner leaflet. The lipid asymmetricities both in the intracellular vesicle membrane and the plasma membrane contribute to synaptic transmission functions. In this study, we developed a cell-sized asymmetric lipid vesicle system containing small-sized asymmetric lipid vesicles (of diameter 200-1000 nm) (asymmetric vesicles-in-a-vesicle), emulating lipid components in the plasma membrane and intracellular vesicle membrane of eukaryotic cells, using microfluidic technology. We successfully constructed an artificial exocytosis system using the asymmetric vesicles-in-a-vesicle system. This asymmetric vesicles-in-avesicle system will be helpful in understanding the mechanisms of vesicle transport, such as neurotransmission and exocytosis.

Received 19th June 2019

Accepted 18th September 2019

DOI: $10.1039 / c 9 r a 04622 d$

rsc.li/rsc-advances

In this study, using an improved pulsed jet-flow method, ${ }^{13,14}$ we directly prepare cell-sized asymmetric lipid vesicles containing asymmetric lipid vesicles (asymmetric vesicles-in-a-vesicle) to emulate asymmetric lipid distribution in the plasma membrane and intracellular vesicle membrane of eukaryotic cells (Fig. 1(a)). The cell-sized vesicles containing vesicles are generated by applying pulsed jet flow against two parallel planar asymmetric lipid bilayers formed in a triple-well device. First, we optimize the width of the second well in the triple-well device and the application time of the jet flow to enclose the small vesicles within the cell-sized lipid vesicles, with two planar lipid bilayers. Next, the asymmetry of the cell-sized lipid vesicles containing the vesicles generated using the triple-well device is examined by confocal laser scanning microscopy (CLSM). Finally, using the asymmetric vesicles-in-a-vesicle system, we demonstrate the fusion of the inner vesicles with the giant vesicles by introducing calcium ions using biological nanopores.

\section{Experimental}

\section{Reagents}

1,2-Dioleoyl-sn-glycero-3-phosphocholine (DOPC), 1,2-dioleoylsn-glycero-3-phospho-L-serine (DOPS), 1,2-dioleoyl-sn-glycero-3phosphoethanolamine (DOPE), 1,2-dioleoyl-sn-glycero-3-phosphoethanolamine- $N$-(lissamine rhodamine B sulfonyl) (RhDOPE), and 7-nitro-2-1,3-benzoxadiazol-4-yl (NBD)-DOPE were purchased from Avanti Polar Lipids, Inc. (Alabaster, AL, USA). Annexin V (Alexa Fluor 488-conjugated) was purchased from Thermo Fisher Scientific (Waltham, MA, USA). $\alpha$-Hemolysin from Staphylococcus aureus was purchased from Sigma-Aldrich Co. LLC (St. Louis, MO, USA).
${ }^{a}$ Artificial Cell Membrane Systems Group, Kanagawa Institute of Industrial Science and Technology, 3-2-1 Sakado, Takatsu-ku, Kawasaki, Kanagawa 213-0012, Japan

${ }^{b}$ Department of Mechano-Informatics, Graduate School of Information Science and Technology, The University of Tokyo, 7-3-1 Hongo, Bunkyo-ku, Tokyo, 113-8656, Japan.E-mail: takeuchi@hybrid.t.u-tokyo.ac.jp

'Division of Molecular Science, Graduate School of Science and Technology, Gunma University, 1-5-1 Tenjin-cho, Kiryu, Gunma 376-8515, Japan 
(a)

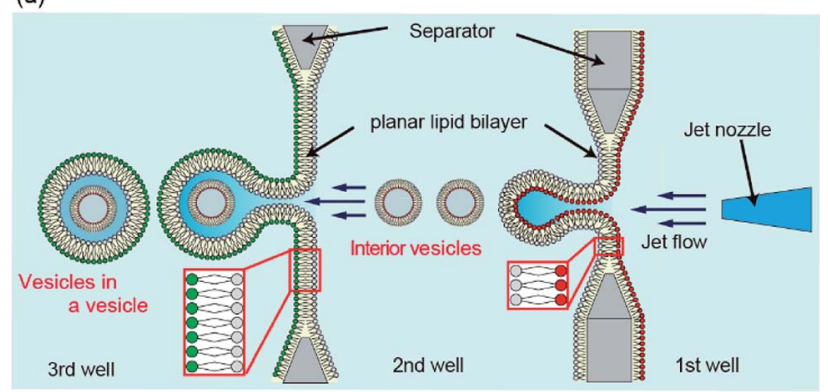

(b)

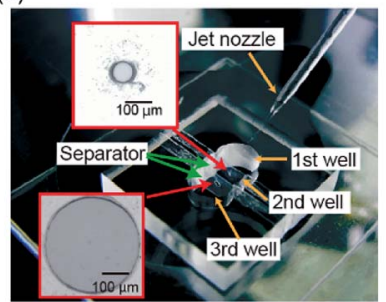

(c)

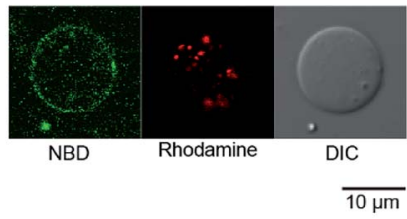

Fig. 1 (a) Schematic representation of generation of vesicles-in-avesicle system. (b) Image of the fabricated device for generating vesicles-in-a-vesicle system. Separators in this device had apertures of the following two sizes: $100 \mu \mathrm{m}$ in diameter between the first and second wells and $500 \mu \mathrm{m}$ in diameter between the second and third wells. (c) A typical confocal image of the vesicles-in-a-vesicle system containing 7-nitro-2-1,3-benzoxadiazol-4-yl (NBD)-lipids in giant vesicle membranes and rhodamine-lipids in the small vesicle.

\section{Device fabrication}

The triple-well device for the generation of vesicles-in-a-vesicle system was fabricated by assembling a poly(methyl methacrylate) (PMMA) plate (Acrylight; Mitsubishi Rayon Co., Ltd., Tokyo, Japan) using an automated computer-aided design and computer-aided manufacturing (CAD/CAM) modeling machine (Model MM-100; Modia Systems Co., Inc., Koshigaya, Japan). Well separators, each with one hole of 100 or $500 \mu \mathrm{m}$ diameter, were prepared from a thin $(75 \mu \mathrm{m})$ PMMA film and a separator with a single hole was mounted between the wells. The chamber was then attached to a flat, $1 \mathrm{~mm}$-thick acrylic substrate by thermocompression bonding. A micro-jet nozzle with a $60 \mu \mathrm{m}$ inner-diameter orifice was formed using a thin-walled glass capillary (G-100; Narishige, Tokyo, Japan) with $1 \mathrm{~mm}$ outer diameter and a micropipette puller (PC-10; Narishige); the nozzle tip was curved $30^{\circ}$ using a microforge (MF-900; Narishige).

\section{Formation of the vesicles-in-a-vesicle system}

Phospholipids (DOPC, DOPS, DOPE, Rh-DOPE, and NBD-DOPE) dissolved in chloroform were evaporated in a glass test tube. Then, $60 \mathrm{mM}$ phospholipid solution was prepared from the lipid film dissolved in $n$-decane solution. All vesicle formations were conducted by $60 \mathrm{mM}$ phospholipid solution. Double planar asymmetric lipid bilayers were formed using the droplet contact method. First, $4 \mu \mathrm{L}$ of DOPC solution containing $0.02 \mathrm{~mol} \% \mathrm{Rh}$-DOPE was added into the first well, $2 \mu \mathrm{L}$ of DOPC/DOPS (1:1 mol ratio) solution was added into the second well, and $4 \mu \mathrm{L}$ of DOPC solution containing $0.02 \mathrm{~mol} \%$ 7-nitro-2-1,3-benzoxadiazol-4-yl (NBD)-DOPE was added into the third well. Next, $19 \mu \mathrm{L}$ of phosphate-buffered saline (PBS; $10 \mathrm{mM} \mathrm{Na}_{2} \mathrm{HPO}_{4} / \mathrm{NaH}_{2} \mathrm{PO}_{4}, 137 \mathrm{mM} \mathrm{NaCl}$, and $2.7 \mathrm{mM} \mathrm{KCl}$; $\mathrm{pH}$ 7.4) containing $500 \mathrm{mM}$ glucose was added to the first well. Eight microliters and $19 \mu \mathrm{L}$ of PBS containing $500 \mathrm{mM}$ sucrose were added into the second and third wells, respectively. A liquid dispensing system was used to control the application time (3.2 $\mathrm{ms})$ and the pressure of the pulsed jet flow (300 kPa) (SJVC3000; Sanei Tech, Japan). The asymmetric vesicles-in-avesicle system was observed by CLSM (FV-1200; Olympus, Tokyo, Japan) with an oil-immersion lens $(\times 60)$ using a diode laser $(559 \mathrm{~nm})$ for rhodamine $(570-670 \mathrm{~nm})$ and a diode laser $(473 \mathrm{~nm})$ for NBD $(485-554 \mathrm{~nm})$.

\section{NBD quenching assay}

An asymmetric vesicles-in-a-vesicle system containing $0.02 \mathrm{~mol} \%$ NBD-DOPE in the outer leaflet of a giant vesicle was prepared using the asymmetry assay. Eight microliters of the vesicle solution collected from the vesicle-generation well was added to $40 \mu \mathrm{L}$ of PBS containing $500 \mathrm{mM}$ glucose. Then, $2 \mu \mathrm{L}$ of $80 \mathrm{mM}$ sodium hydrosulfite was added to the outer solution of asymmetric vesicles. The generated asymmetric vesicles-in-avesicle system was observed by CLSM with an oil-immersion lens $(\times 60)$ using a diode laser $(473 \mathrm{~nm})$ for NBD (485-554 $\mathrm{nm})$. Fluorescence intensities were measured using image $\mathrm{J}$ (NIH, USA).

\section{Annexin V assay}

Asymmetric vesicles-in-a-vesicle systems were prepared using lipid solutions of (i) DOPC (1st and 3rd wells) and DOPS/DOPE (9 : $1 \mathrm{~mol} \%$ ) (2nd well) or (ii) DOPC (2nd well) and DOPS/DOPE (9 : $1 \mathrm{~mol} \%$ ) (1st and 3rd wells). Next, $19 \mu \mathrm{L}$ of HEPES buffer (10 mM HEPES and $140 \mathrm{mM} \mathrm{NaCl}$; pH 7.4) containing $500 \mathrm{mM}$ glucose was added to the first well. Six microliters of HEPES buffer containing $500 \mathrm{mM}$ sucrose, $1 \mu \mathrm{L}$ of 5 -fold diluted Alexa Fluor 488-conjugated Annexin V solution, and $1 \mu \mathrm{L}$ of $8 \mathrm{mM}$ calcium ions were added to the second well. Nineteen microliters of HEPES buffer containing $500 \mathrm{mM}$ sucrose was added to the third well. The asymmetric vesicles-in-a-vesicle was then generated by the pulsed jet flow. Alexa Fluor 488 in the asymmetric vesicles-in-a-vesicle was observed by CLSM with an oilimmersion lens $(\times 60)$ using a diode laser $(473 \mathrm{~nm})$ for Alexa Fluor 488 (485-554 nm). Fluorescence intensities were measured using image $\mathrm{J}$ (NIH, USA).

\section{Observation of fusion of small vesicles into the giant vesicle}

An asymmetric vesicles-in-a-vesicle system was prepared using lipid solutions of DOPC (3rd well), DOPC containing $0.02 \mathrm{~mol} \%$ Rh-DOPE (1st well), and DOPC/DOPS/DOPE (4 : $5: 1 \mathrm{~mol} \%$ ) or DOPC/DOPS (1:1 mol\%) (2nd well). Next, $19 \mu \mathrm{L}$ of HEPES buffer (10 mM HEPES and $140 \mathrm{mM} \mathrm{NaCl}$; pH 7.4) containing $500 \mathrm{mM}$ glucose was added to the first well. Eight microliters and $19 \mu \mathrm{L}$ of HEPES buffer containing $500 \mathrm{mM}$ sucrose were added to the second and third wells, respectively. The asymmetric vesicles-in-a-vesicle was then generated using pulsed jet 
flow. Eight microliters of vesicle solution collected from the vesicle-generation well was added to $36 \mu \mathrm{L}$ of HEPES buffer containing $500 \mathrm{mM}$ glucose. Then, $1 \mu \mathrm{L}$ of $30 \mu \mathrm{M} \alpha$-hemolysin and $5 \mu \mathrm{L}$ of $50 \mathrm{mM} \mathrm{CaCl}_{2}$ were added to the outer solution of the asymmetric vesicles. The fusion of asymmetric vesicles-in-avesicle was observed by CLSM with an oil-immersion lens $(\times 60)$ using a diode laser $(559 \mathrm{~nm})$ for rhodamine $(570-670 \mathrm{~nm})$.

\section{Results and discussion}

\section{Formation of the vesicles-in-a-vesicle system}

We previously reported that the size distribution of giant lipid vesicles formed using the pulsed jet-flow method could be regulated by changing the area of the planar lipid bilayer and the application time of the jet flow. ${ }^{\mathbf{1 3}}$ For instance, when the area of the planar lipid bilayer is increased, the vesicle diameter increases. When the application time of the jet flow is increased, the vesicle diameter decreases. Based on these results, to generate giant vesicles containing smaller vesicles, that emulate asymmetric lipid distribution in the plasma membrane and intracellular vesicle membrane in eukaryotic cells, we developed a triple-well device that mounts two separators with apertures of diameter $100 \mu \mathrm{m}$ (to generate inner vesicles) between the first and second wells and $500 \mu \mathrm{m}$ in diameter (to generate cell-sized vesicles) between the second and third wells (Fig. 1(a) and (b)). The lipid monolayer of the second well in the triple well-device was shared by the outer leaflet of the inner vesicles and the inner leaflet of the giant vesicles. The asymmetric lipid distribution in the vesicle membrane is a crucial feature of the triple device.

The giant vesicles containing the smaller vesicles were generated by adjusting the width of the second well and the application time and pressure of the jet flow. When the vesiclesin-a-vesicle system was generated using the triple-well device with the width of the second well of $1.8 \mathrm{~mm}$, the giant vesicles containing the smaller vesicles were not observed. We determined that the optimal width of the second well is $1.2 \mathrm{~mm}$, application time of the jet flow is $3.2 \mathrm{~ms}$, and pressure of the jet flow is $300 \mathrm{kPa}$. The results suggested that the vesicles-in-avesicle system was generated from each planar lipid bilayer. Under the abovementioned conditions (i.e., jet flow application time: $3.2 \mathrm{~ms}$ and application pressure: $300 \mathrm{kPa}$ ), the generation ratio of cell-sized vesicles containing the smaller vesicles was approximately $18 \%$ (5/28, three explements). The generation ratio of the vesicles-in-a-vesicle system can be improved by modifying the size of the planar lipid bilayer, diameter of the jet nozzle, and pressure of the jet flow. The average diameter of the giant vesicles was $23.6 \pm 9.2 \mu \mathrm{m}$. On the contrary, the diameter range of the inner vesicles within the giant vesicles was approximately 200-1000 $\mathrm{nm}$.

\section{Asymmetry of the vesicles-in-a-vesicle system}

To confirm the asymmetry of the giant vesicle membranes containing the smaller vesicles, we conducted fluorescence quenching of phospholipid-conjugated NBD in the outer leaflet of the giant vesicles. Sodium hydrosulfite was added to the outer solution of the giant vesicles. NBD in the outer leaflet of the vesicles was only quenched by sodium hydrosulfite. ${ }^{15}$ Fig. 2(a) shows typical confocal microscopy images of the smaller vesicle-containing asymmetric giant vesicles, generated from the second planar lipid bilayer with $0.02 \mathrm{~mol} \% \mathrm{NBD}$-conjugated DOPE/DOPC in the outer leaflet, before and after the addition of sodium hydrosulfite. NBD fluorescence on the giant vesicle membranes significantly decreased within $10 \mathrm{~min}$. The retention rate of NBD fluorescence was approximately $5.5 \% \pm 5.3 \%$. The retention rate
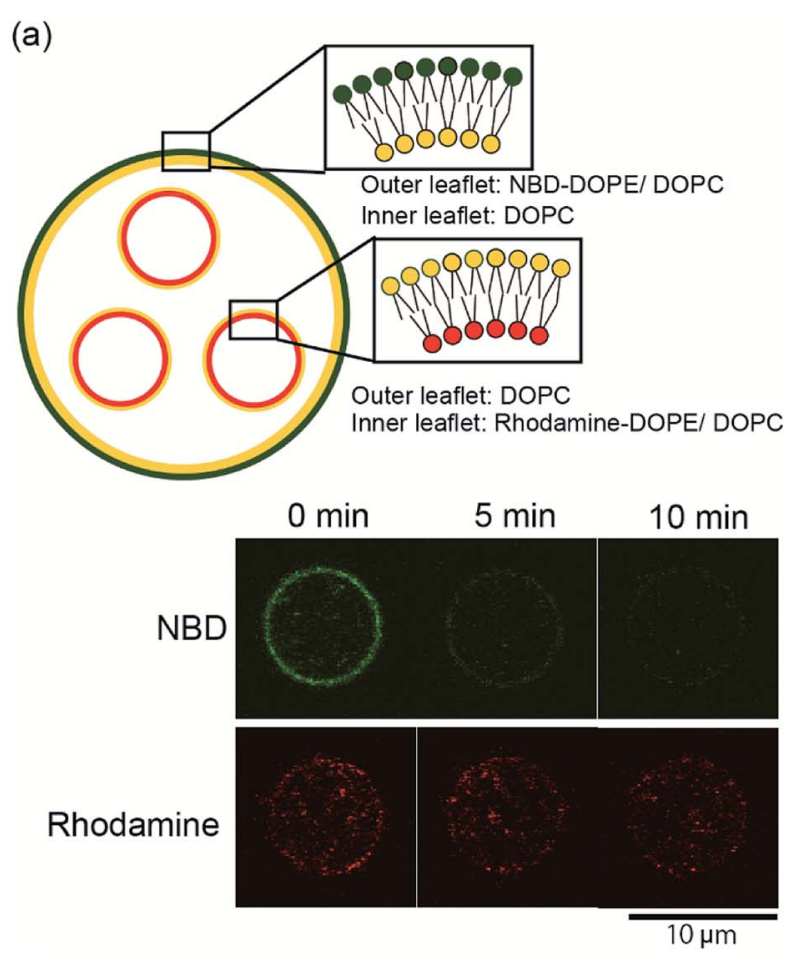

(b)

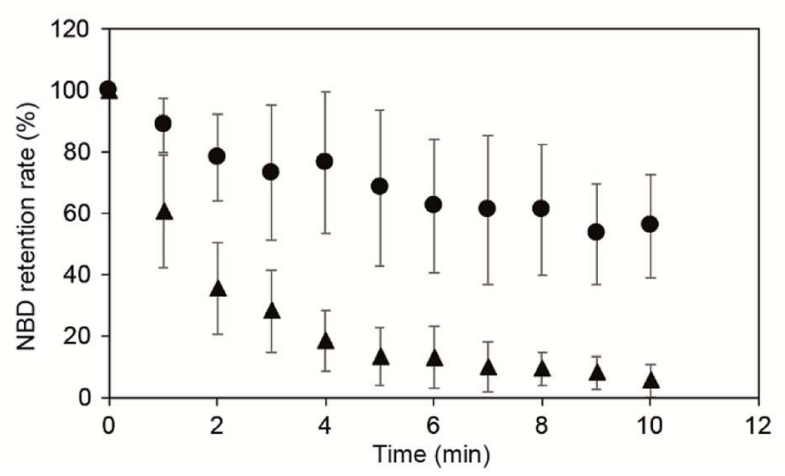

Fig. 2 (a) Schematic representation of components of the asymmetric vesicles-in-a-vesicle system used for the fluorescence-quenching assay. The vesicles-in-a-vesicle system consisted of NBD-conjugated lipids in the outer leaflet of the giant vesicle. Typical time-lapse images of the vesicles-in-a-vesicle system obtained using the fluorescencequenching assay. (b) Fluorescence intensities determined using the NBD-quenching assay. The graph shows the time-lapse of the NBDquenching ratio of the asymmetric vesicles-in-a-vesicle (triangles) ( $n$ $=13$ ) and symmetric vesicles-in-a-vesicle system with NBD-lipids on both leaflets (circles) $(n=10)$. Error bars, standard deviations. 
of NBD fluorescence on the symmetric giant vesicles was approximately $55.9 \% \pm 16.9 \%$ (Fig. 2(b)). These results suggest that our device can be used to generate giant vesicles containing small vesicles with asymmetric lipid membranes.

Next, we investigated the asymmetry of the inner leaflet of the giant vesicles and the outer leaflet of the inner vesicles using the fluorescent Annexin V binding assay. Annexin V was specifically bound to PS in the presence of calcium ions. ${ }^{16} \mathrm{We}$ added Annexin $\mathrm{V}$ and calcium chloride to the second well containing the asymmetric vesicles-in-a-vesicle system. We generated giant asymmetric vesicles containing smaller vesicles using the following two methods: (i) DOPC solution was added to the first and third wells and DOPS/DOPE (9:1 mol\%) solution was added to the second well and (ii) DOPC solution was added to the second well and DOPS/DOPE ( $9: 1 \mathrm{~mol} \%$ ) solution was added to the first and third wells. Consequently, we obtained (i) giant asymmetric vesicles encapsulating smaller asymmetric vesicles composed of DOPS/DOPE (9:1 mol\%) in the outer leaflet and DOPC in the inner leaflet and (ii) giant asymmetric vesicles and small

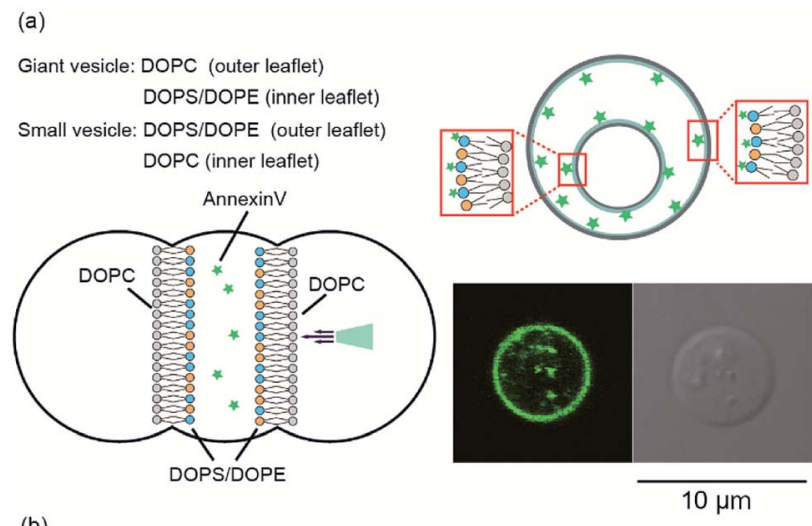

(b)

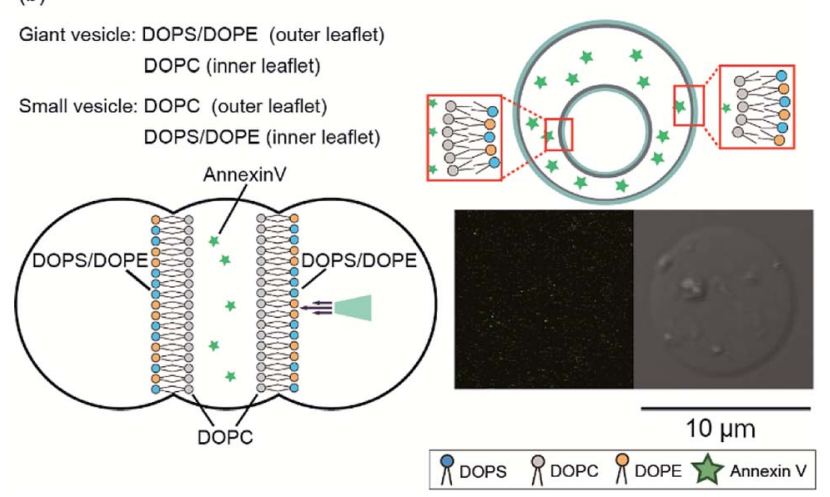

Fig. 3 Investigation of asymmetry of the inner leaflet of the giant vesicles and the outer leaflet of the inner vesicles using fluorescent Annexin V. (a) Asymmetric vesicles-in-a-vesicle system composed of DOPC in the outer leaflet and DOPS/DOPE (9:1 mol\%) in the inner leaflet of the giant vesicles and of DOPS/DOPE ( $9: 1 \mathrm{~mol} \%)$ in the outer leaflet and DOPC in the inner leaflet of the inner vesicles. (b) Asymmetric vesicles-in-a-vesicle composed of DOPS/DOPE (9:1 mol\%) in the outer leaflet and DOPC in the inner leaflet of the giant vesicles and of DOPC in the outer leaflet and DOPS/DOPE (9:1 mol\%) in the inner leaflet of the inner vesicles. vesicles with opposite compositions, respectively. Fig. 3 shows the schematics and typical confocal microscopy images of the giant asymmetric vesicles containing smaller asymmetric vesicles with the lipid compositions (i) and (ii). Annexin V fluorescence was observed on the giant and inner vesicle membranes composed of DOPC in the outer leaflet of the giant vesicles and the inner leaflet of inner vesicles, and of DOPS/DOPE (9:1 mol\%) in the inner leaflet of giant vesicles and the outer leaflet of inner vesicles (lipid composition (i)) (Fig. 3(a)). Annexin V fluorescence on the asymmetric giant vesicles containing the smaller vesicles with lipid composition (ii) was not observed (Fig. 3(b)). The detection ratio of Annexin V-incorporated fluorescence on both giant vesicle membranes and inner vesicle membranes was $72.4 \%$. Therefore, using this system, we generated giant asymmetric vesicles containing asymmetric vesicles to mimic asymmetric lipid distribution of eukaryotic cells for first time. $^{6}$

\section{Fusion of the inner vesicles in the asymmetric vesicles-in-a- vesicle system}

We constructed an induction system for artificial exocytosis by calcium ion influx into giant vesicles. We prepared an asymmetric vesicles-in-a-vesicle system; the giant asymmetric vesicles were composed of DOPC in the outer leaflet and DOPC/DOPS/DOPE (4:5:1 mol\%) in the inner leaflet, and the inner asymmetric vesicles were composed of DOPC/DOPS/ DOPE in the outer leaflet and DOPC containing $0.02 \mathrm{~mol} \%$ Rh-DOPE in the inner leaflet. The fusion of giant asymmetric vesicles and inner asymmetric vesicles was induced by calcium ion influx through nanopores into asymmetric giant vesicles. We added $0.6 \mu \mathrm{M}$ (final concentration) nanopores $(\alpha$ hemolysin) and $5 \mathrm{mM}$ (final concentration) calcium ions to the outer solution of asymmetric giant vesicles. After approximately 15-20 min, we observed rhodamine fluorescence on the asymmetric giant vesicle membranes (Fig. 4(a)). The fluorescence of the inner rhodamine-vesicles was decreased in the asymmetric giant vesicles, while the rhodamine fluorescence intensity was increased on the giant vesicle surfaces (Fig. 4(c)). This result indicated that the inner rhodamine vesicles were fused to the membranes of the asymmetric giant vesicles. On the contrary, the fusion phenomenon of an asymmetric vesicles-in-a vesicle without DOPE (DOPC/DOPS (1:1 mol\%)) in the inner leaflet of the giant vesicle and outer leaflet of the inner vesicles was not observed (Fig. 4(b)). This result indicated that the presence of DOPE might contribute to the promotion of vesicle fusion. The extracellular transport of the biomolecules via the inner (intracellular) vesicles into the biological cells was caused by membrane fusion between the intracellular vesicle membrane and plasma membrane in the presence of calcium ions, PS, PE and the membrane protein soluble $\mathrm{N}$ ethylmaleimide-sensitive factor attachment protein receptors (SNARE). ${ }^{17}$ Our exocytosis system emulates the exocytosis system of eukaryotic cells in terms of membrane fusion induced by calcium ions and asymmetric lipid distribution. 
(a) With DOPE

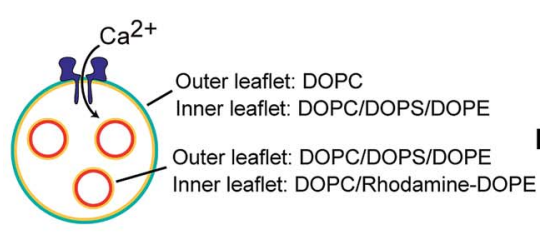

(b) Without DOPE

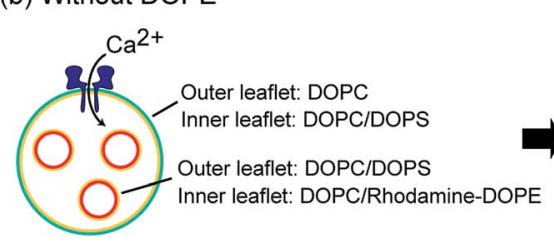

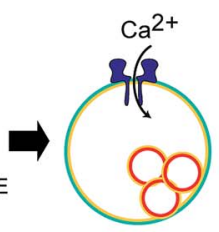
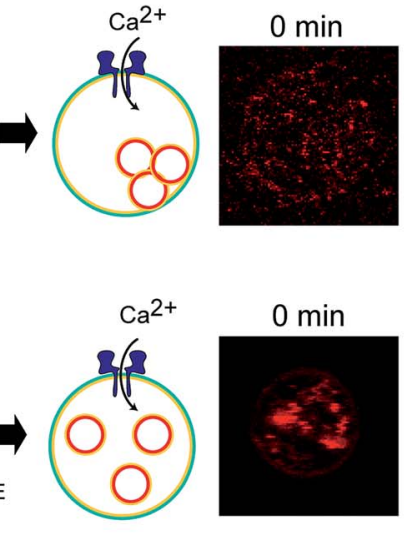
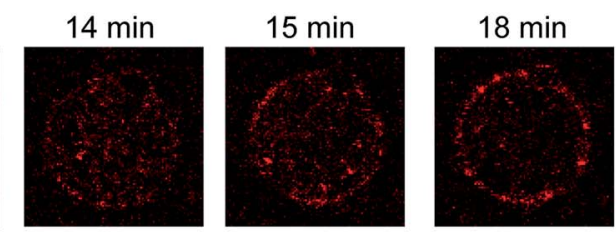

$10 \mu \mathrm{m}$

(c)

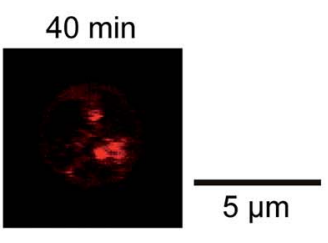

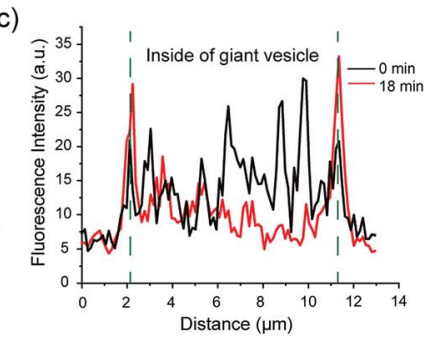

Fig. 4 Demonstration of vesicular fusion of intracellular vesicles using the asymmetric vesicles-in-a-vesicle system. (a) Asymmetric vesicles-ina-vesicle system with DOPE in the inner leaflet of the giant vesicles and the outer leaflet of the inner vesicles. (b) Asymmetric vesicles-in-avesicle system without DOPE. (c) Line profiles of rhodamine fluorescence intensity of asymmetric vesicles-in-a-vesicle system with DOPE in the inner leaflet of the giant vesicles and the outer leaflet of the inner vesicles. The black and red lines show the rhodamine fluorescence before and after calcium ion influx, respectively. The green-dashed lines represent the surface of giant vesicles.

\section{Conclusions}

Although the fusion of symmetric lipid vesicles has been reported, ${ }^{\mathbf{9} 10}$ this is the first observation of fusion between giant asymmetric vesicles and asymmetric vesicles within giant asymmetric vesicles. This vesicle fusion system, which mimics the fusion phenomenon of intracellular vesicles, will be useful for investigating models of intracellular vesicular traffic, such as autophagy or exosomes. In artificial cell studies, a bioreactor for releasing medicines by external input, such as ions, heat, and light, can be developed using this vesicles-in-a-vesicle system in the future. Moreover, the use of this asymmetric vesicles-in-avesicle system will help understanding of mechanisms of vesicle transport, such as neurotransmission and exocytosis.

\section{Conflicts of interest}

There are no conflicts to declare.

\section{Acknowledgements}

We acknowledge the technical support provided by Ms Y. Nozaki. This work was partly supported by a Grant-in-Aid for Scientific Research (B) (JP18H02092 K. K.) from the Japan Society for the Promotion of Science (JSPS) and the Regional Innovation Strategy Support Program of the Ministry of Education, Culture, Sports, Science and Technology (MEXT), Japan.

\section{Notes and references}

1 A. Herrmann and P. Miiller, Biosci. Rep., 1986, 6, 185-191.
2 G. van Meer, D. R. Voelker and G. W. Feigenson, Nat. Rev. Mol. Cell Biol., 2008, 9, 112-124.

3 H. I. Ingólfsson, M. N. Melo, F. J. van Eerden, C. Arnarez, C. A. Lopez, T. A. Wassenaar, X. Periole, A. H. de Vries, D. P. Tieleman and S. J. Marrink, J. Am. Chem. Soc., 2014, 136, 14554-14559.

4 T. Harayama and H. Riezman, Nat. Rev. Mol. Cell Biol., 2018, 19, 281-296.

5 C. de Duve, Nat. Rev. Genet., 2007, 8, 395-403.

6 D. L. Daleke, J. Biol. Chem., 2007, 282, 821-825.

7 R. Panatala, H. Hennrich and J. C. M. Holthuis, J. Cell Sci., 2015, 128, 2021-2032.

8 C. L. Jackson, L. Walch and J.-M. Verbavatz, Dev. Cell, 2016, 39, 139-153.

9 M. Sasai, S. Tadokoro and N. Hirashima, Langmuir, 2010, 26, 14788-14792.

10 M. Hadorn, E. Boenzli, P. Eggenberger Hotz and M. M. Hanczyc, PLoS One, 2012, 7, 1-7.

11 P.-Y. Bolinger, D. Stamou and H. Vogel, Angew. Chem., Int. Ed., 2008, 47, 5544-5549.

12 J. W. Hindley, Y. Elani, C. M. McGilvery, S. Ali, C. L. Bevan, R. V. Law and O. Ces, Nat. Commun., 2018, 9, 1093.

13 K. Kamiya, R. Kawano, T. Osaki, K. Akiyoshi and S. Takeuchi, Nat. Chem., 2016, 8, 881-889.

14 K. Kamiya and S. Takeuchi, J. Mater. Chem. B, 2017, 5, 59115923.

15 S. Pautot, B. J. Frisken, D. A. Weitz and T. C. Lubensky, Proc. Natl. Acad. Sci. U. S. A., 2003, 100, 10718-10721.

16 P. C. Hu, S. Li and N. Malmstadt, ACS Appl. Mater. Interfaces, 2011, 3, 1434-1440.

17 D. Tareste, J. Shen, T. J. Melia and J. E. Rothman, Proc. Natl. Acad. Sci. U. S. A., 2008, 105, 2380-2385. 